\title{
Remotely Controlled in Situ Growth of Silver Microwires Forming Bioelectronic Interfaces
}

\author{
Paola Sanjuan-Alberte, ${ }^{\dagger+}$ Ehab Saleh, ${ }^{\ddagger}$ Andie J. Shaw, ${ }^{\dagger, \ddagger}$ Nicola Lacalendola, ${ }^{\prime \prime}$ Geoff Willmott, ${ }^{\| \odot}$ \\ Jayasheelan Vaithilingam, ${ }^{\ddagger}$ Morgan R. Alexander, ${ }^{\S}$ Richard J. M. Hague, ${ }^{\ddagger}$ and Frankie J. Rawson*,†॰
}

${ }^{\dagger}$ Regenerative Medicine and Cellular Therapies, School of Pharmacy, ${ }^{\ddagger}$ Centre for Additive Manufacturing, Faculty of Engineering, and ${ }^{\S}$ Advanced Materials and Healthcare Technologies, School of Pharmacy, University of Nottingham, Nottingham NG7 2RD, U.K.

${ }^{\|}$The MacDiarmid Institute for Advanced Materials and Technology, Department of Physics and Chemistry, The University of Auckland, Auckland 1010, New Zealand

Supporting Information

ABSTRACT: There is a pressing need to advance our ability to construct three-dimensional (3D) functional bioelectronic interfaces. Additionally, to ease the transition to building cellular electronic systems, a remote approach to merge electrical components with biology is desirable. By combining 3D digital inkjet printing with bipolar electrochemistry, we remotely control and fabricate conductive wires, forming a first of its kind contactless bionic manufacturing procedure. It enables controlled fabrication of conductive wires in a three-dimensional configuration. Moreover, we demonstrate that this technology could be used to grow and interface conductive conduits in situ with mammalian cells, offering a new strategy to engineering bioelectronic interfaces. This represents a step change in the

production of functional complex circuitry and considerably increases the manufacturing capabilities of merging cells with electronics. This approach provides a platform to construct bioelectronics in situ offering a potential paradigm shift in the methods for building bioelectronics with potential applications in biosensing and bioelectronic medicine.

KEYWORDS: bioelectronic interfaces, microwire growth, bipolar electrochemistry, inkjet printing, bionic manufacturing

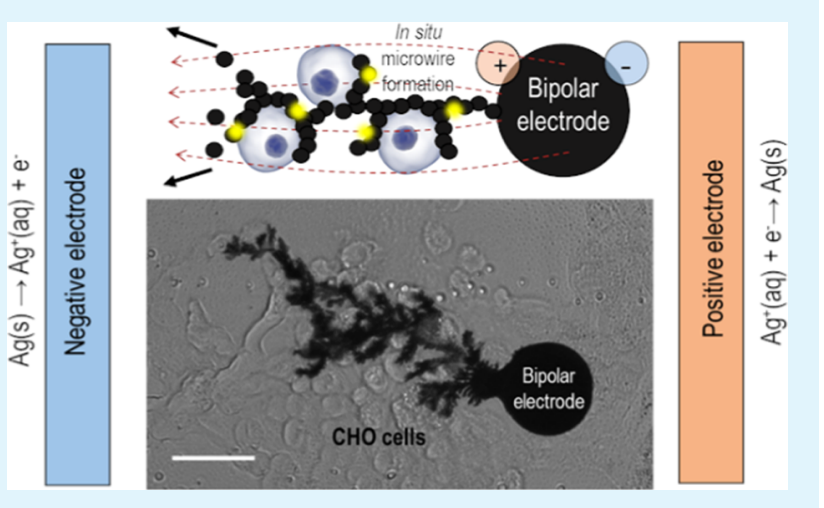

\section{INTRODUCTION}

Bioelectronics is the merging of biology with electronics, and current state-of-the-art bioelectronics devices are most prevalently preassembled prior to integration with biology. This poses issues in the development of bioelectronic systems, since to work optimally they require seamless integration between electronic and biological structures to facilitate the two-way communication between the biotic and abiotic electrical interfaces. ${ }^{1}$ Such connectivity is currently achieved in diverse fields such as bioelectronic medicine, ${ }^{2}$ optogenetics, ${ }^{3}$ biosensing, ${ }^{4}$ and microbial fuel cells. ${ }^{5}$ However, there have been limited examples of seamless integration of electronic components with the biology, and current methods tend to be highly invasive. To reduce the invasiveness, electronic elements should ideally have nanoscale features and be biocompatible and softer than biological components. ${ }^{6}$ Although considerable advances in materials are being accomplished, ${ }^{7-10}$ manufacturing strategies represent a key step to achieve fully integrated devices. $^{11}$

Nanotechnological approaches such as nanofield effect transistors $^{12}$ or nanowires ${ }^{13,14}$ have yielded significant improvements in sensitivity and biocompatibility, ${ }^{15}$ and there 


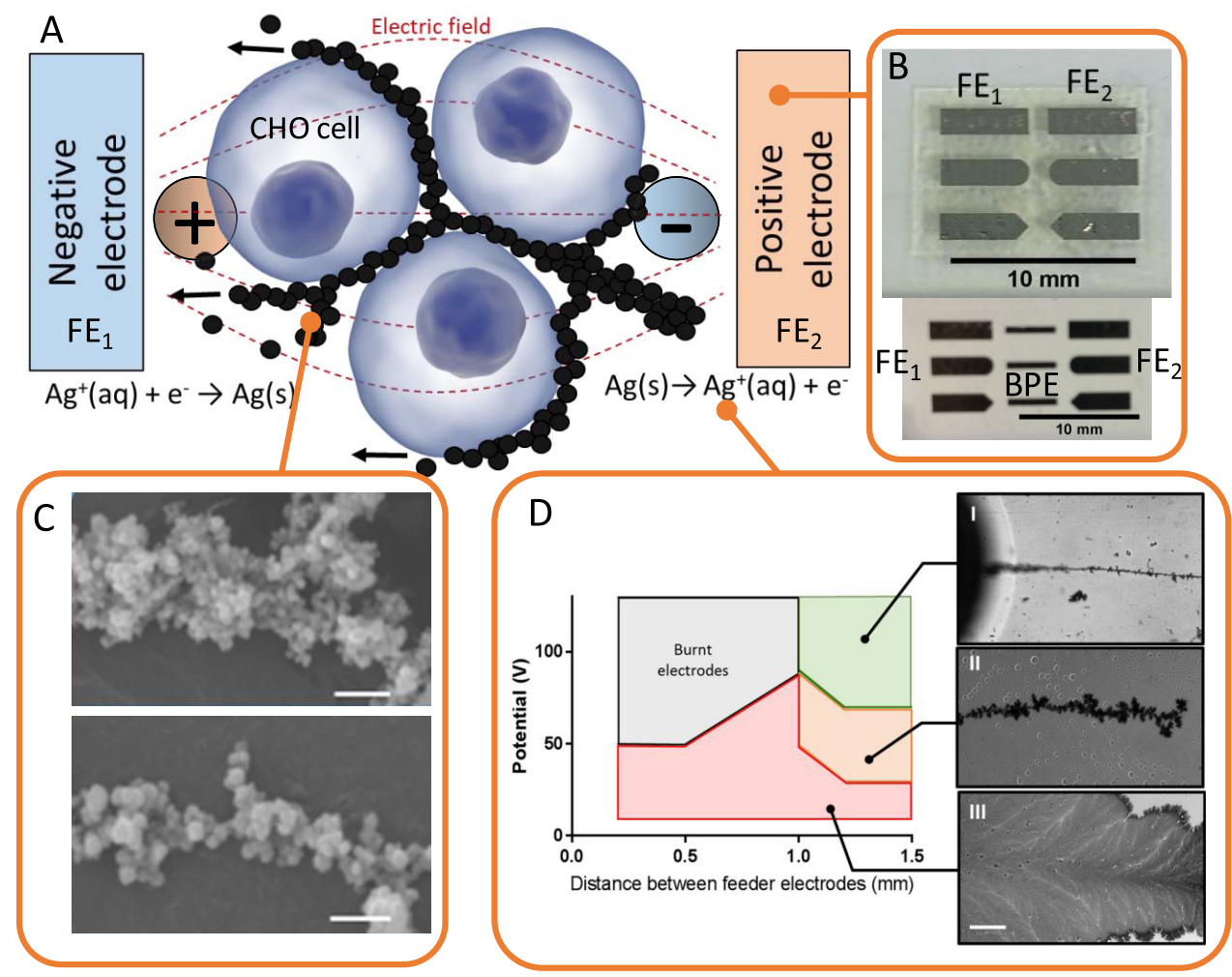

Figure 1. Mechanisms of wire growth. (A) Schematic of bioelectronic interfacial formations between CHO cells and self-assembled silver nanoparticles when a potential difference is applied between silver feeder electrodes $\left(\mathrm{FE}_{1}\right.$ and $\left.\mathrm{FE}_{2}\right)$. (B) Inkjet-printed silver feeder electrodes separated by $1 \mathrm{~mm}$ and bipolar electrodes (BPEs). (C) Scanning electron microscopy (SEM) of self-assembled silver nanoparticles resulting in different diameters. Scale bar $1 \mu \mathrm{m}$. (D) Optimization of potentials applied (V) vs distance between feeder electrodes (mm) to induce microwire growth resulting in (I) thin microwires, (II) thick microwires, and (III) deposition at the surface. Scale bar $100 \mu \mathrm{m}$.

allow for the hierarchical integration of multifunctional materials in 3D combined with biology. ${ }^{24}$ This has been successfully achieved by printing a combination of living chondrocytes and an inductive coil antenna to produce a bionic ear with an enhanced auditory sensing. ${ }^{25}$

To minituarize and control remotely the manufacturing of bioelectronic components in situ, a combination of additive manufacturing and wireless electrochemistry was studied. Wireless electrochemistry, also known as bipolar electrochemistry, has been used in diverse areas which include the generation of self-powered structures or microswimmers, ${ }^{26}$ sensing methods, ${ }^{27}$ and production of Janus objects. ${ }^{28}$ However, there are no known reports of its use in the formation of cellular bioelectronic systems. Controlled growth of micro- ${ }^{29}$ and nanowires ${ }^{30}$ and electrical contacts between particles $^{31}$ have been previously reported by this method.

One-dimensional nanostructures with label-free biosensing capabilities have been developed by directional growth techniques, where changes in conductance across conductive polymers $^{32}$ or metallic nanowires ${ }^{33,34}$ in response to molecular binding can be detected. ${ }^{35}$ Nanostructures are generated by electropolymerization or electrodeposition of metals and directed between two electrodes patterned by lithographic methods. ${ }^{33}$ This approach has been used to generate conductive nanostructures between Dictyostelium cells, the gap between the electrodes was limited to $<30 \mu \mathrm{m}$ and could not be controlled remotely, limiting its bioelectronic applications. $^{36}$

The aim of the work presented herein is to develop new bionic manufacturing techniques to merge conductive materials with biology (Figure 1A). A key objective to achieving this was to overcome these limitations by combining the design freedom offered by inkjet printing with bipolar electrochemistry. This was achieved by (i) designing an electrochemical cell and optimizing microwire (MW) growth and formation; (ii) testing the functionality of wires formed by powering of a light-emitting diode (LED); and (iii) interfacing the microwires with Chinese hamster ovary $(\mathrm{CHO})$ cells and testing the sensing capabilities of the system. Thereby, in the first demonstration of its kind, we present a contactless method of creating bioelectronic functional systems in situ. This represents a step change in the production of complex circuitry and considerably increases the manufacturing capabilities of merging cells with electronics. Bioelectronic interfaces formed were controlled remotely, giving rise to a new strategy that may avoid invasive surgery typically required in a bioelectronic medicine.

\section{RESULTS AND DISCUSSION}

2.1. Inkjet Printing Electrochemical Cells. Our initial approach to the controlled fabrication of 3D electronic systems was to develop a printed electrochemical cell that enabled the growth of conducting conduits using bipolar electrochemistry in a controlled 3D manner. Toward this goal, a pair of silver electrodes (feeder electrodes) (Figure 1B) were printed using a drop-on-demand inkjet printer. A silver nanoparticle-infused ink (AgNP ink) was used to print feeder and bipolar electrodes, as its electrochemical behavior can be taken as an advantage of wirelessly grown conductive conduits in a single step without the need for the addition of metallic salts. 
A
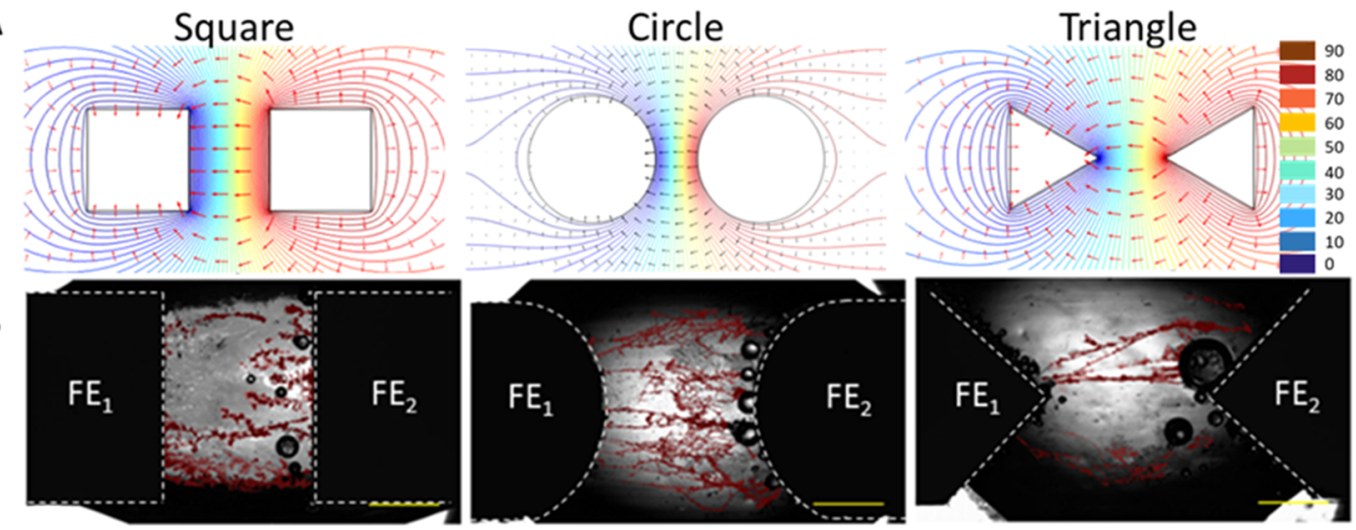

C
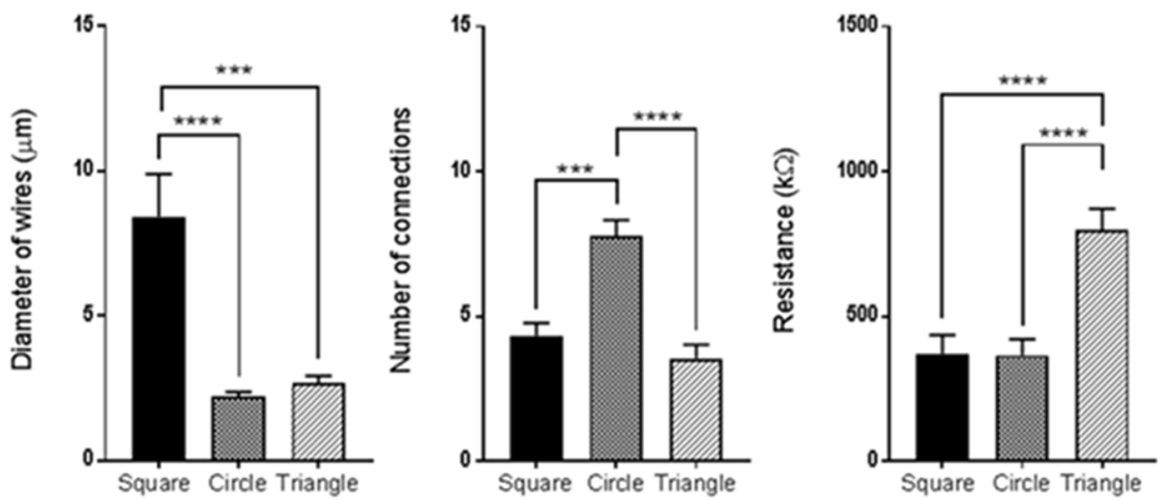

Figure 2. Growth of silver microwires (AgMW) with varied geometry of feeder electrode (FE). (A) Modeled electric field and electronic potential of $90 \mathrm{~V}$ applied using different geometries of feeder electrodes (contour: electric potential (V), arrow surface: electric field). (B) Optical microscopy images of AgMW connections between feeder electrodes in the shapes of square, circle, and triangle. AgMWs were false colored in red to highlight them. Scale bar $500 \mu \mathrm{m}$. (C) Diameter, number of connections, and resistance of wires established between electrodes at different geometric shaped feeder electrodes. Error bars represent standard error mean, further statistical analysis was performed using one-way analysis of variance (ANOVA) with Tukey's honestly significant difference post hoc analysis $(n=9 ; p \leq 0.0001)$.

Furthermore, it is suitably conductive and has ideal printing properties that result in it being one of the most used metals in inkjet printing for electronic applications. ${ }^{37}$ It is important to note that the material used is not a key point of this work, but that the materials used do provide the ability to combine conductive and biological structures remotely. Details of the optimization of the printing process and the characterization of printed patterns can be found in the Supporting Information.

2.2. Characterization of Electrochemically Deposited Silver Microwires. The next part of the investigation was to optimize the mechanism of silver microwire (AgMW)-induced metal deposition, which would be used later in electrochemical wireless studies to produce functional bioelectronic systems. When a potential difference is applied to silver feeder electrodes separated by an aqueous phase, the negative electrode oxidizes silver atoms on its surface, making them available in the solution as silver cations (Figure 1A). The positive electrode, hence, reduces these cations depositing them onto its surface as it follows

Negative electrode:

$$
\operatorname{Ag}(\mathrm{s})-\mathrm{e}^{-} \rightarrow \operatorname{Ag}^{+}(\mathrm{aq})
$$

Positive electrode:

$$
\operatorname{Ag}^{+}(\mathrm{aq})+\mathrm{e}^{-} \rightarrow \operatorname{Ag}(\mathrm{s})
$$

Magnitude and kinetics of redox reactions depend on the potentials applied and the electric field intensity. ${ }^{38}$ Parameters were selected to optimize the conditions for electrodeposition of silver. This was achieved by studying the deposits formed as a function of potential applied and distance between feeder electrodes. At distances $<1 \mathrm{~mm}$ between feeder electrodes and potentials above $50 \mathrm{~V}$, the reactions were too violent due to too much current being passed, resulting in burnt feeder electrodes (Figure 1D). Lower potentials induced an unorganized deposition that formed a thin film on the surface of the tripropyleneglycol diacrylate (TPGDA). Distances $>1 \mathrm{~mm}$ between feeder electrodes at 30-60 V applied induced an organized reduction of silver in the shape of silver microwires (AgMWs). Magnitudes of potentials above $70 \mathrm{~V}$ made thinner wires compared to those where relatively lower potentials were applied (Figure 1D). These differences responded to the magnitude of energy required to induce the redox reactions and the kinetics of the reactions. Therefore, higher values of potentials supply enough energy to the system to form organized structures in contrast to lower potentials.

Once the conditions were optimized, a potential difference of $90 \mathrm{~V}$ was applied in pulses of $300 \mu$ s over a period of $10 \mathrm{~s}$ through feeder electrodes with a separation of $1 \mathrm{~mm}$. This induced the reduction of silver cations and self-assembly of AgMWs at the positive electrode (Figure 1A). The morphology of the AgMWs was characterized by light microscopy and scanning electron microscopy (SEM). Energy dispersive X-ray (EDX) spectroscopic images confirmed that silver was the predominant element in the wires (Figure S3), and the expected electrochemical reactions were taking place 
A

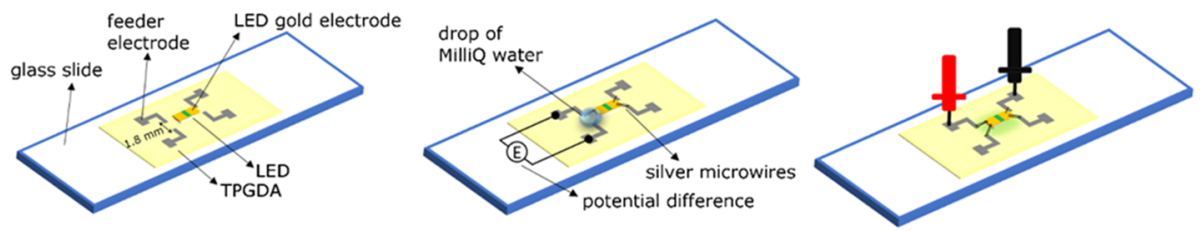

B
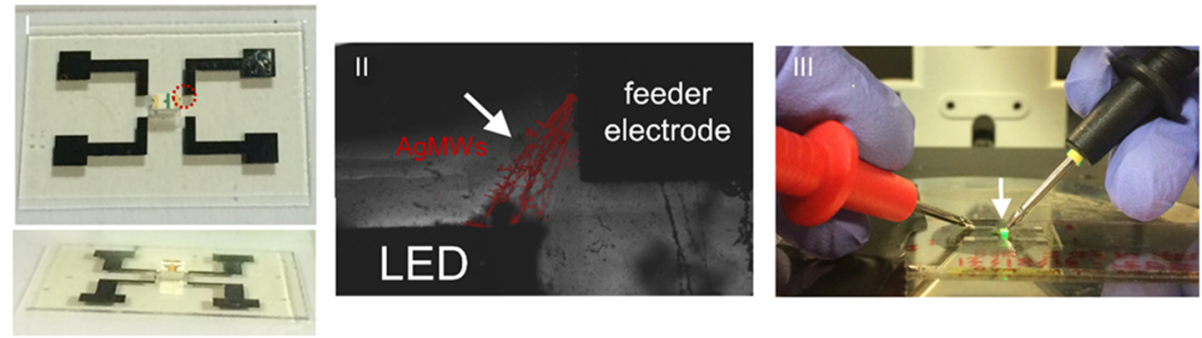

Figure 3. Multimaterial three-dimensional device. (A) Schematic representation of a fabrication process. (B) (I) Top and frontal views of printed electrodes and placement of a light-emitting diode, (II) optical microscope image of a magnified area within the red circle in (I) showing AgMWs forming a 3D connection with one of the gold electrodes of the LED, AgMWs were false colored in red and pointed with an arrow; and (III) test of functionality indicating green diode light (arrow).

within our printed system. Regarding the unorganized film, random distribution of silver can be observed in the EDX images. Further examination of the microwires using SEM revealed clusters of deposited silver nanoparticles (NPs). The mechanism that results in the formation of silver nanoparticle is not currently clear. However, this does not affect our understanding of how they form wires or the application for which we use later. According to SEM images of wires formed, silver-electrodeposited NPs had an average size of $212 \pm 48$ $\mathrm{nm}(n=25)$. The assembly between these particles is affected by the magnitude of the potential, which influences the diameter of the AgMWs (Figure 1D), with a minimum diameter equivalent to the size of single NPs (Figure 1C).

Electric field strength is determined by the applied potential difference and electrode dimensions. Increases in the strength of the applied electric field affect the migration of ions within the solution and, thus, the rate of assembly of silver particles. Therefore, our ability to control the electrical field characteristics is of significant importance in terms of influencing AgMW growth. ${ }^{31,38}$ Simulation images of electric fields and potentials of feeder electrodes with different geometries can be seen in Figure 2A, showing the effect of geometry on resulting electric fields. In this work, three simple geometries (square, circle, and triangle) of feeder electrodes were tested under the conditions mentioned above. AgMW assembly was observed in situ and in real time by optical microscopy (Figure 2B). Wire growth matched the simulated electric field lines from Figure 2A. Some bubbles produced by electrochemical water splitting can be seen near the feeder electrodes $\left(\mathrm{FE}_{2}\right)$, however, they do not have an effect in the microwire formation.

AgMWs are more polarizable than the surrounding dielectric medium, being subjected to the dielectrophoretic force that induces growth in the direction of increasing electric field strength. In the square electrodes, dendritic wires with a mean diameter of $8.402 \pm 1.483 \mu \mathrm{m}$ are formed (Figure $2 \mathrm{C}$ ). This is likely due to the Joule heating and thermal fluctuations produced, interfering with the field alignment and producing the dendrites. Circular electrodes distribute the field more evenly, producing a larger number of thinner wires (7.78 \pm 0.55 wires with a diameter of $2.216 \pm 0.158 \mu \mathrm{m}$ on average), whereas triangular electrodes produced sharp and thin AgMWs with a mean diameter of $2.70 \pm 0.23 \mu \mathrm{m}$. The number and diameter of wires also influence the resistance measured. Higher values $880 \pm 67 \mathrm{k} \Omega$ are found on the triangular electrodes, in contrast to $372 \pm 65$ and $366 \pm 56 \mathrm{k} \Omega$ in the square and circular electrodes, respectively. The growth and electrical properties of AgMW can thus be easily modified by changes in the geometry of the feeder electrodes, which can be adjusted depending on the applications pursued. However, the values of resistance obtained are different from theoretical values calculated using the very well-known formula of electrical resistance (eq 3 ), assuming a minimum diameter of the cross-section of single wires of $200 \mathrm{~nm}$ which provides the maximum value of resistance theoretically expected (5.0525 $\Omega)$.

$$
R=\rho \frac{L}{A}
$$

where $R$ represents resistance, $\rho$ the material resistivity, $L$ the length of the wire, and $A$ its cross-section. A thickness of 200 $\mathrm{nm}$ was selected as it is the minimum thickness that we have seen in the SEM images (Figure 1C) and according to the equation, it should give us the maximum value of resistance we should observe within our system in optimal conditions.

As the geometry of feeder electrodes affects AgMW growth, the addition of varying geometry bipolar electrodes (BPEs) was also evaluated. Square-, circular-, triangular-, and diamondshaped BPEs were printed between circular feeder electrodes. The area of the BPEs was controlled to $1.5 \mathrm{~mm}^{2}$, with the gap between feeder electrodes being $2.5 \mathrm{~mm}$ and the BPE positioned in the center (Figure S4). The addition of the $\mathrm{BPE}$ required a higher potential difference of $120 \mathrm{~V}$ to be applied; again, this was applied in pulses of $300 \mu \mathrm{s}$. BPE shape altered the electric field and hence affected wire growth direction. Circular and square electrodes grew AgMWs uniformly from each side of the BPE, whereas triangular and diamond BPEs grew wires to and from their vertices. As seen in Figure S4, wire diameter, number of connections, and wire resistance were evaluated. Although wire diameter was not significantly different between geometries ranging between 3.36 and $5.7 \mu \mathrm{m}$, diamond BPEs grew significantly more wires than both circular and triangular BPEs. Resistance was also 

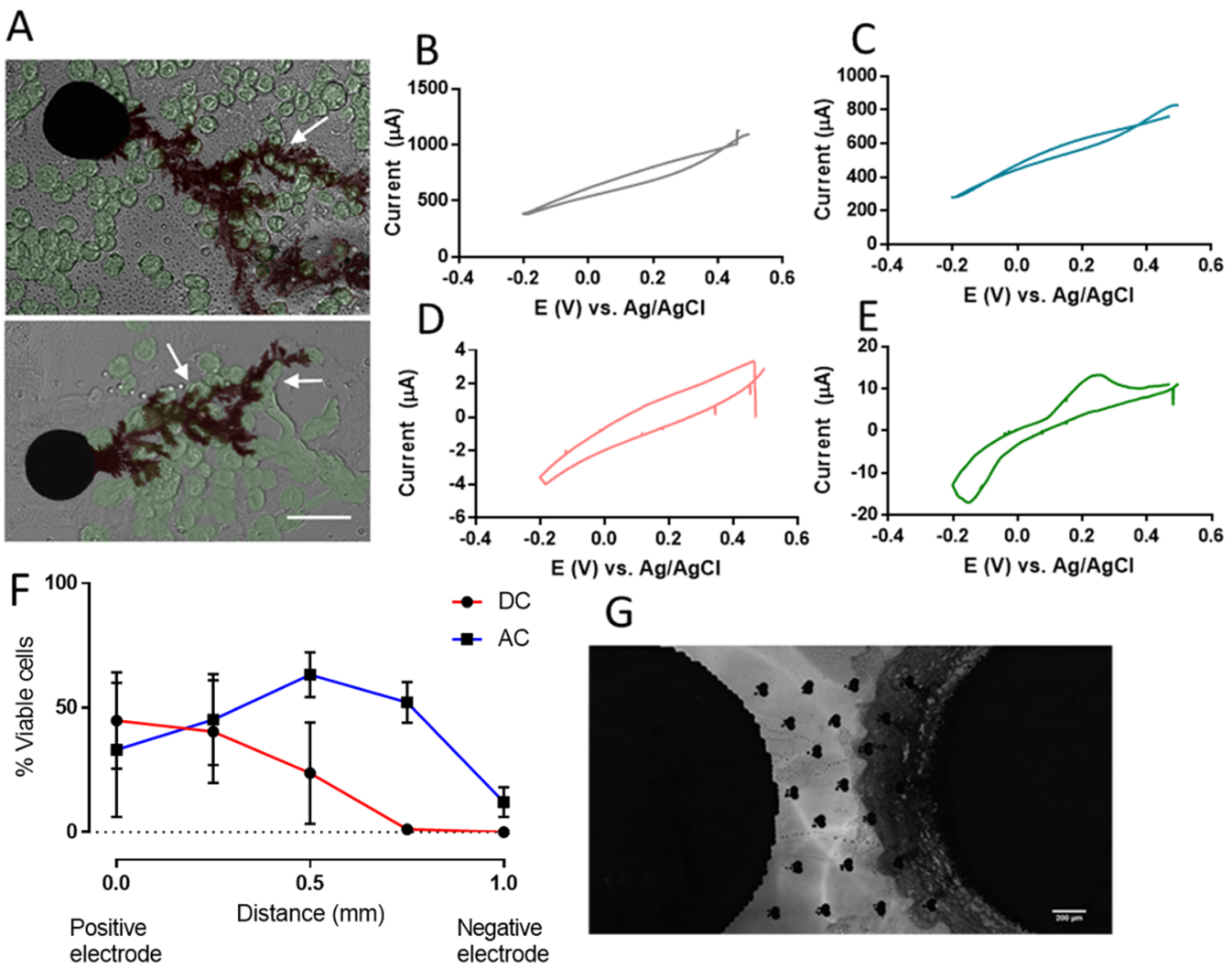

Figure 4. In situ formation of bioelectronics interfaces. (A) Optical microscope image of AgMWs (red) growing between CHO cells (green). AgMWs form a conductive mesh surrounding cells (arrows). Scale bar $50 \mu \mathrm{m}$. Circles of silver with a diameter of $67 \pm 6.2 \mu \mathrm{m}$ were printed between the feeder electrodes to guide silver wires acting as bipolar electrodes (BPEs). Typical cyclic voltammograms (CVs) obtained (B) before (left) and (C) after AgMW growth in the absence of cells and (D) before and (E) after AgMW growth in the presence of cells. Potentials were applied from 0.5 to $-0.2 \mathrm{~V}$ at a scan rate $100 \mathrm{mV} \mathrm{s}^{-1}$. Phosphate-buffered saline (PBS) dissolved in Milli-Q water was used as a supporting electrolyte. Mean values of three different samples were calculated and represented within the CVs. (F) Percentage of viable cells after wire growth using direct current (DC) relative to the percentage of viable cells prior to potential application with direct current (DC) and alternating current (AC) $(N=3, n=3$; standard deviation (SD)). (G) Optical microscopy image of CHO cells after trypan blue viability assay after DC application of $90 \mathrm{~V}$.

similar between geometries ranging from 673.67 to $871.83 \mathrm{k} \Omega$; the significant difference in resistance between square and circle BPEs is thought to be due to the slightly larger area of the square BPE.

The ability to control AgMW growth was also studied through altering feeder electrode and BPE geometry and forming different patterns which directed wires along a path. This can be seen in Figure S5.

\subsection{Wireless Electrochemical Fabrication of Func-} tional 3D Circuitry. Subsequent experiments were aimed at demonstrating that controlled growth of AgMW connecting conduits in a functional electronic 3D-circuit could be achieved. The principle of this was to use wireless electrochemistry to control and direct growth of wires from a lightemitting diode to the 3D inkjet-printed electronic system, thereby completing the circuit. Dimensions and construction details can be found in the experimental details. Two pairs of square feeder electrodes were inkjet-printed (Figure 3A). This geometry was chosen due to the low-resistance values and improved stability of AgMWs, as compared to wires grown at other feeder electrode geometries due to increased wire thickness (Figure 2B). A light-emitting diode (LED) was placed upside-down between the two pairs, leaving a gap in the $z$-plane of $500 \mu \mathrm{m}$ between the gold electrodes of the LED and the substrate and, thus, the silver feeder electrodes (Figure 3B).

A potential difference of $150 \mathrm{~V}$ was applied in an aqueous environment without any gold or silver cations present in solution prior to applying a potential. The polarization of the gold electrode occurs when a threshold potential is applied. The gold electrodes are renowned for being inert so no redox associated with gold would occur. The polarization of the feeder electrodes causes silver redox leading to the AgMW formation between feeder electrodes, and wires grew to and from the surface of the LED at an angle of approximately $25^{\circ}$ relative to the glass surface based on the geometry, configuring three-dimensional interconnects between the elements of the system.

2.4. Fabricating Bioelectronic Tools by Combining Inkjet Printing with Wireless Electrochemistry. Although we have demonstrated the ability to couple wireless electrochemistry with inkjet printing to fabricate $3 \mathrm{D}$ electronic functional systems, it was also apparent that such technology could be important in future bioelectronic applications. Consequently, we investigated the ability to use the combined wireless electrochemistry enabled by inkjet printing to form $3 \mathrm{D}$ conductive cellular conduits in situ without any physical electrical connection, with the aim to potentially use this to sense cell behavior. To demonstrate this, Chinese hamster 
ovary $(\mathrm{CHO})$ cells were cultured and placed between feeder electrodes. When a potential of $90 \mathrm{~V}$ was applied, a mesh of conductive conduit-cellular constructs was formed (Figure 4A). These wires surrounded the cells resulting in wire growth adapting to the shape of the cells. This is due to the higher resistance of the cellular membrane to ionic currents induced by external electric fields, ${ }^{39}$ leading to a microwire growth to areas of lower resistance and guiding the process. Circles of silver with a dimension of $67 \pm 6.2 \mu \mathrm{m}$ were printed between the feeder electrodes to act as BPEs and facilitate guidance of AgMW growth, reducing the circuit resistance created by the cells (Figure S6).

Cyclic voltammetry was performed to elucidate the ability of the wirelessly formed bioelectronic system, via cellular-conduit connects to sense biological redox events. Cyclic voltammograms (CVs) were performed with printed electrodes using a starting potential of $0.5 \mathrm{~V}$, a switching potential of $-0.2 \mathrm{~V}$, and an end potential of $0.5 \mathrm{~V}$ at a scan rate of $100 \mathrm{mV} \mathrm{s}^{-1}$ with PBS as an electrolyte. These CVs were performed prior to and after wireless growth of AgMWs in the absence and presence of cells. Redox peaks were observed in the CVs in the presence of the AgMWs and the cells (Figure 4E), with a mean potential peak observed at $-0.130 \pm 0.022 \mathrm{~V}( \pm 1 \mathrm{SD} n=3)$ and at $0.279 \pm 0.047 \mathrm{~V}( \pm 1 \mathrm{SD} n=3)$, for the reduction and oxidation events, respectively. We conclude that this observed redox behavior is of biological origin as no resulting redox peaks were observed in CVs without the cells (Figure 4B,C). There are two possibilities that give rise to the redox behavior, however, both are biologically driven because the redox behavior is only observed in the presence of the cells and wires after application of potentials. First, the redox behavior represents silver electrochemistry which is induced by an interaction of the cells with the electrode. Second, the redox peaks observed represent a cell molecule or redox interaction with the cells. In both the cases, bio-functionality of the system is observed as the cells presence is required to detect the signal obtained in the presence of wires. It is interesting to observe that when we compare cyclic voltammograms in the absence and in the presence of cells, the magnitude of the current in the presence of the cells is around 3 orders of magnitude smaller. This occurs as the cells increase the circuit resistance and hinder electron transfer.

Trypan blue viability assay was performed to elucidate the percentage of viable cells prior to and after microwire growth. Prior to application of potentials, $98.9 \%$ of viable cells was found at all points between feeder electrodes. To identify the effect of potential application and discard effects on viability due to material and solvents, this value was assumed as $100 \%$ in our next calculations. After the application of $90 \mathrm{~V}$ using direct current, it is possible to observe a pronounced decay on the values of viability in areas next to the negative electrode (Figure 4F). Cells are attracted to the negative electrode due to the effects of ionic current, and in areas near this electrode, the cell mortality is higher (Figure 4G). In areas near the positive electrode, the viability is higher (44.9\%), however, the cell number is more reduced. Cell viability was also studied when potentials were applied using alternating current (AC), resulting in a parabolic curve where higher values of viability were seen in the center of the area between the feeder electrodes (63.3\%) and lower viability values were seen in areas closer to the electrodes (Figure $4 \mathrm{~F}$ ). When the areas under the curve of both graphs were compared (21.95 \pm 5.67 in $\mathrm{DC}$ vs $45.82 \pm 5.20$ in $\mathrm{AC}$ ), it can be seen that cell viability is higher after exposure to AC electrical inputs. From this, it can be concluded that the nature of the electric input applied can have an effect on cell viability. Cytotoxicity associated to silver could also have an effect on low viability seen, and more biocompatible materials such as conducting polymers could be an alternative to solve this, however, current techniques are not compatible with biology due to the nature of the solvents used in these processes. ${ }^{29}$

\section{CONCLUSIONS}

Our approach based on the combination of both inkjet printing and wireless electrochemistry resulted in the production of conductive conduits of silver which could be used to build novel 3D multimaterial, multicomponent functional bioelectronic systems remotely. Wires were formed by clusters of electrodeposited silver nanoparticles and showed a dependence of electric field applied tuned via feeder electrode geometry. Thus, this technological advancement provides a promising fabrication platform to develop innovative 3D multifunctional multimaterial bioelectronic systems with potential applications in biosensing, bioelectronic medicine, and fabrication of 3D circuits.

\section{MATERIALS AND METHODS}

4.1. Materials. Glass slides $\left(76 \times 26 \times 1 \mathrm{~mm}^{3}\right.$, Cole-Parmer $)$ were used as substrates and washed with acetone and isopropanol prior to use. Inks consisted of a silver nanoparticle (AgNP ink) with an approximate $30 \%$ of solid content purchased from Advanced Nano Products Ltd. Tripropyleneglycol diacrylate (TPGDA) ink was synthesized in-house. A method describing the preparation of this ink was published elsewhere. ${ }^{40}$ Surface mount Osram Opto LG R971, CHIPLED 0805 Series Green 570 nm light-emitting diodes (LEDs), and conductive silver paste were purchased from RS Components. Ethanol HPLC grade, 3-(trimethoxysilyl) propyl methacrylate (TMSPMA) 98\%, toluene anhydrous 99.8\%, Dulbecco's modified Eagles high glucose medium, (4-(2-hydroxyethyl)-1-piperazineethanesulfonic acid) (HEPES) solution $1 \mathrm{M}, \mathrm{pH}$ 7.0-7.6; fetal bovine serum (FBS), trypsin solution, penicillin/streptomycin solution, trypan blue, and phosphate-buffered saline (PBS) in tablets were acquired from Sigma-Aldrich.

4.2. Inkjet Printing Process. The instrument used was a piezoelectric drop-on-demand Dimatix Materials Printer (Model: DMP-2800, FUJIFILM, Dimatix, Inc. Santa Clara, CA). Inks were filtered (HPLC Nylon $5.0 \mu \mathrm{m}$ syringe filters, Cole-Parmer) before injecting into the propylene print cartridge (DMC-11610, $10 \mathrm{pl}$ ), which was fixed to a print head consisting of 16 nozzles $(21 \mu \mathrm{m}$ in diameter). Bitmap images of patterns to be printed were designed by the GNU Image Manipulation Program (version 2.8.16) and adapted using the Dimatix materials printer software. The print resolution was 846.67 drops per inch, corresponding to a drop spacing of $30 \mu \mathrm{m}$, and using custom print waveforms. The firing voltage varied over a range of 24-30 V. Droplet ejection was monitored using a camera incorporated in the printer.

To avoid any curing inside the cartridge when printing TPGDA due to ambient light, the cartridge was masked with duct tape. Five layers of TPGDA were printed and cured with a UV light $(365 \mathrm{~nm}$ and $6000 \mathrm{~mJ} \mathrm{~cm}^{-2}$ ) mounted to the printing unit allowing it to move with the print head and induce real-time UV illumination and curing (Printed Electronics Ltd.).

Three layers of AgNP ink were printed on top of the five layers of TPGDA to improve the adhesion of the silver to the substrate, achieving a good level of conductivity. A single nozzle was used. The glass substrates were heated using an in-house built heating system inside the printing platform and held at $200{ }^{\circ} \mathrm{C}$ during the printing process to enable solvent evaporation and thermal sintering.

4.3. Surface Analysis. Roughness profile of one layer of printed silver on $1 \mu \mathrm{m}^{2}$ areas and thickness on $20 \mu \mathrm{m}^{2}$ areas were established 
by atomic force microscopy (AFM) using a Dimension FastScan AFM system (Bruker Corporation) in peak force mode mapping in air at room temperature. Probes consisted of a RTESPA MPP-11120-10 model with a cantilever size of 3.5-4.5 $\mu \mathrm{m}$ and a frequency of 347$393 \mathrm{kHz}$. Data analysis of the AFM images was performed using WSxM 5.0 and Gwyddion 2.43 software.

4.4. Resistance Measurements. Resistance of inkjet-printed silver surfaces was measured using a $25 \mathrm{kHz}$ LCR Meter HM8018 (Rhode \& Schwartz) in $2 \times 2 \mathrm{~mm}^{2}$ squares varying the temperature and time of sintering and number of layers printed and sintering time $(n=10)$. Small droplets of silver paste were deposited over the ends of the printed silver layers to prevent scratches from the contact with the probes of the LCR Meter, allowing them to dry at room temperature for $10 \mathrm{~min}$. Data was processed in GraphPad PRISM version 7.01 .

4.5. Microwire Growth. Micorwires were grown by applying a potential difference of $90 \mathrm{~V}$ in the presence of aqueous electrolyte using an in-house-made high-voltage pulsed power supply in 10 pulses of $300 \mathrm{~ms}$ with a gap between pulses of $200 \mathrm{~ms}$ for a duration of $10 \mathrm{~s}$. Different geometries of electrodes were tested. The separation between the electrodes was $1 \mathrm{~mm}$.

Different geometries were also tested using bipolar electrodes with different shapes with an area of length of $1.5 \mathrm{~mm}^{2}$ was printed between the feeder electrodes. The separation between feeder electrodes was $2 \mathrm{~mm}$. A potential of $120 \mathrm{~V}$ was applied as described in the previous paragraph. Values of resistance were measured $(n=9)$ using a digital multimeter (Eurisem Technics, model EP719). Number and diameter of connections were obtained after the application of potential differences $(n=9)$. Wires were only counted if they formed a continuous connection between the feeder electrodes. Diameter was measured in wires connected to the negative feeder electrode (effective interconnections) ( $n=10$ per sample). Data was analyzed using the GraphPad PRISM. A D'Agostino \& Pearson normality test was carried out to check Gaussian distribution, and one-way ANOVA with Tukey's post analysis was carried out to compare data between groups.

Digital imaging microcopy was performed using a Nikon ECLIPSE TE300 fluorescent microscope equipped with a QImaging optiMOS, Scientific CMOS camera. The number of frames acquired per second were 20. Electron dispersive X-ray (EDX) of uncoated wires was performed in a Hitachi TM 3000 tabletop scanning electron microscope. Scanning electron microscopy (SEM) images were obtained in a JEOL 6060LV at $20 \mathrm{kV}$. Wires were deposited on SEM pin stubs and coated with gold.

4.6. Computer Modeling of Electric Fields. Finite element method modeling was carried out using the COMSOL Multiphysics 5.2 with the Electrostatics package. In each simulation, electrodes are separated by $1 \mathrm{~mm}$, and the size of each electrode (side length or diameter) was $1.5 \mathrm{~mm}$. A positive potential of $90 \mathrm{~V}$ was applied to the left electrode, and the right acts as a ground. Contour lines indicate the field intensity, whereas the arrows indicate the direction of the field.

4.7. Multicomponent Functional Electronic Device Formation. Two pairs of silver electrodes, with an area of $10.29 \mathrm{~mm}^{2}$ each, were printed. Each pair of electrodes had a separation of 1.8 $\mathrm{mm}$, and the separation between each pair was $2.6 \mathrm{~mm}$. A surface mount light-emitting diode (LED) was placed upside-down between the two pairs of printed electrodes at a distance of $0.4 \mathrm{~mm}$. LED was attached to the surface with a drop of TPGDA and cured under UV light for $10 \mathrm{~s}$. Deionized water $(10 \mu \mathrm{L})$ was deposited partially covering the two extremes of one pair of the printed silver electrodes and one Au electrode of the LED. A continuous potential difference of $150 \mathrm{~V}$ was applied over the pair of printed silver electrodes during $15 \mathrm{~s}$, inducing the microwire formation. This was repeated on the other pair of electrodes. The functionality of the system was tested using a multimeter in a diode test mode.

4.8. Bioelectronic System Fabrication. Glass slides were chemically modified before the printing process to increase the adhesion of TPGDA to glass substrate over longer periods of time. Glass was cleaned in ethanol during 10 min prior to $\mathrm{O}_{2}$ plasma- etching. Glass slides were then placed into the plasma chamber between two copper band electrodes connected to a power source (Coaxial Power Ltd.). The chamber was evacuated, and needle valves (BOC Edwards) were used to control the oxygen pressure. Pressure was monitored with a Pirani gauge (Kurt J. Lesker Ltd.), and a glowdischarged plasma was initiated when the pressure was stabilized to 9 $\times 10^{-2}$ Mbar ( $50 \mathrm{~W}$ for $3 \mathrm{~min}$ ). Cleaned glass slides were then silanized in 1\% TMSPMA in toluene for an hour and washed in MilliQ water.

CHO cells were cultured in corning T-75 $\mathrm{cm}^{2}$ flasks in $15 \mathrm{~mL}$ of media (supplemented with 10\% FBS, 5\% HEPES solution, and $1 \%$ penicillin/streptomycin) for $2-3$ days in an incubator at $37{ }^{\circ} \mathrm{C}$ in $5 \%$ $\mathrm{CO}_{2}$ atmosphere and passaged when $70-80 \%$ confluent. A stickyslide well (Ibidi, Martinsried, Germany) with a growth area per well of $1 \mathrm{~cm}^{2}$ was attached to a glass slide where silver electrodes were previously printed. Electrodes had $1 \mathrm{~mm}$ separation between them, and $67 \pm 6.2 \mu \mathrm{m}$ diameter circles were randomly printed between them to facilitate microwire growth. Media $(300 \mu \mathrm{L})$ containing approximately $5 \times 10^{5}$ cells was added and cultured for $3 \mathrm{~h}$ at $37^{\circ} \mathrm{C}$ in $5 \% \mathrm{CO}_{2}$ atmosphere. Once the cells were attached to the surface, media was washed with PBS and substituted with Milli-Q water during microwire growth. A potential difference of $90 \mathrm{~V}$ was applied during $10 \mathrm{~s}$.

Electrochemical measurements were carried out at room temperature using a VersaSTAT 4 potentiostat interfaced with a personal computer and integrated with VersaStudio Software (Princeton Applied Research). PBS dissolved in Milli-Q water was used as a supporting electrolyte. Potentials are reported vs an $\mathrm{Ag} / \mathrm{AgCl}$ reference electrode. Printed silver feeder electrodes were used as working electrodes, and a platinum wire was used as an auxiliary electrode. Cyclic voltammogram (CVs) was acquired when a potential was applied from 0.5 to $-0.2 \mathrm{~V}$ at a scan rate of $100 \mathrm{mV} \mathrm{s}^{-1}$. Current was measured in three different samples prior and after AgMW growth in the presence and absence of the cells.

Trypan blue viability assay was performed prior and after wire growth, and percentage of viable cells were counted on three areas of $100 \times 100 \mu \mathrm{m}^{2}$ at each distance point on three diferent samples $(N=$ $3, n=3$; SD). AC potentials were applied at values of $90 \mathrm{~V}$ and $1 \mathrm{~Hz}$ using a Programmable AC/DC power source EC1000SA (NF Corporation).

\section{ASSOCIATED CONTENT}

\section{Supporting Information}

The Supporting Information is available free of charge on the ACS Publications website at DOI: 10.1021/acsami.8b22075.

Characterization of silver inkjet-printed electrodes (speed of droplet ejection, morphology before/after thermal sintering, parameter optimization, and thickness of printed layers); EDX analysis of silver films/wires; microwire growth on bipolar electrodes, bioelectronic interfaces formation (PDF)

\section{AUTHOR INFORMATION}

\section{Corresponding Author}

*E-mail: frankie.rawson@nottingham.ac.uk.

ORCID $\odot$

Geoff Willmott: 0000-0001-5079-2622

Jayasheelan Vaithilingam: 0000-0002-5501-5344

Frankie J. Rawson: 0000-0002-4872-8928

\section{Author Contributions}

P.S.-A. performed printing, optimization, and characterization of wire growth, LED experiments, and cell work. E.S. built the in-house-made high-voltage-pulsed power supply used in the experimental set up and contributed to the experiment design. N.L. and G.W. carried out the simulation of electric fields and 
electric potentials of feeder electrodes with different geometries. A.S. designed and printed patterns with different shapes of BPE and performed wire growth on these samples. P.S.-A. and F.J.R. wrote the manuscript. All authors discussed the experimental results and read, commented, and approved the final manuscript.

\section{Funding}

This work was supported by the Engineering and Physical Sciences Research Council grant numbers [EP/R004072/1, $\mathrm{EP} / \mathrm{K} 005138 / 11$, EP/N006615/1]. The University of Nottingham is thanked for funding Dr Rawson's Nottingham Research Fellowship.

\section{Notes}

The authors declare no competing financial interest.

\section{ACKNOWLEDGMENTS}

The authors would like to acknowledge Dr Marta AlvarezPaino for her assistance with the scanning electron microscopy and Jacqueline M. Hicks for help setting up the fluorescent microscope.

\section{REFERENCES}

(1) Rawson, F. J. New Dimensions in Controlling Cellular Function with Electroceutics. Ther. Delivery 2015, 6, 5-8.

(2) Famm, K.; Litt, B.; Tracey, K. J.; Boyden, E. S.; Slaoui, M. Drug Discovery: a Jump-Start for Electroceuticals. Nature 2013, 496, 159161.

(3) Deisseroth, K. Optogenetics. Nat. Methods 2011, 8, 26-29.

(4) El-Ali, J.; Sorger, P. K.; Jensen, K. F. Cells on Chips. Nature 2006, 442, 403-411.

(5) Logan, B. E. Exoelectrogenic Bacteria that Power Microbial Fuel Cells. Nat. Rev. Microbiol. 2009, 7, 375-381.

(6) Liu, J.; Xie, C.; Dai, X.; Jin, L.; Zhou, W.; Lieber, C. M. Multifunctional Three-Dimensional Macroporous Nanoelectronic Networks for Smart Materials. Proc. Natl. Acad. Sci. U.S.A. 2013, 110, 6694-6699.

(7) Malliaras, G.; Abidian, M. R. Organic Bioelectronic Materials and Devices. Adv. Mater. 2015, 27, 7492.

(8) Akinwande, D.; Petrone, N.; Hone, J. Two-Dimensional Flexible Nanoelectronics. Nat. Commun. 2014, 5, No. 5678.

(9) Zeng, W.; Shu, L.; Li, Q.; Chen, S.; Wang, F.; Tao, X. M. FiberBased Wearable Electronics: a Review of Materials, Fabrication, Devices and Applications. Adv. Mater. 2014, 26, 5310-5336.

(10) Liu, J.; Fu, T.-M.; Cheng, Z.; Hong, G.; Zhou, T.; Jin, L.; Duvvuri, M.; Jiang, Z.; Kruskal, P.; Xie, C.; Suo, Z.; Fang, Y.; Lieber, C. M. Syringe-injectable electronics. Nat. Nanotechnol. 2015, 10, 629-636.

(11) Sanjuan-Alberte, P.; Alexander, M. R.; Hague, R. J. M.; Rawson, F. J. Electrochemically stimulating developments in bioelectronic medicine. Bioelectron. Med. 2018, 4, 1.

(12) Qing, Q.; Jiang, Z.; Xu, L.; Gao, R.; Mai, L.; Lieber, C. M. FreeStanding Kinked Nanowire Transistor Probes for Targeted Intracellular Recording in Three Dimensions. Nat. Nanotechnol. 2013, 9, 142-147.

(13) Carrad, D. J.; Mostert, B.; Meredith, P.; Micolich, A. P. Hybrid Nanowire Ion-to-Electron Transducers for Integrated Bioelectronic Circuitry, Organic Sensors and Bioelectronics IX, 2016; Vol. 9944.

(14) García-Carmona, L.; Moreno-Guzman, M.; Martin, A.; Martinez, S. B.; Fernandez-Martinez, A. B.; Gonzalez, M. C.; LucioCazana, J.; Escarpa, A. Aligned Copper Nanowires as a Cut-and-Paste Exclusive Electrochemical Transducer for Free-Enzyme Highly Selective Quantification of Intracellular Hydrogen Peroxide in Cisplatin-Treated Cells. Biosens. Bioelectron. 2017, 96, 146-151.

(15) Zhang, A.; Lieber, C. M. Nano-Bioelectronics. Chem. Rev. 2016, $116,215-257$.
(16) Kim, T. I.; McCall, J. G.; Jung, Y. H.; Huang, X.; Siuda, E. R.; Li, Y.; Song, J.; Song, Y. M.; Pao, H. A.; Kim, R. H.; Lu, C.; Lee, S. D.; Song, I. S.; Shin, G.; Al-Hasani, R.; Kim, S.; Tan, M. P.; Huang, Y.; Omenetto, F. G.; Rogers, J. A.; Bruchas, M. R. Injectable, CellularScale Optoelectronics with Applications for Wireless Optogenetics. Science 2013, 340, 211-216.

(17) Park, J.; Kim, Y. S.; Ryu, S.; Kang, W. S.; Park, S.; Han, J.; Jeong, H. C.; Hong, B. H.; Ahn, Y.; Kim, B. S. Graphene Potentiates the Myocardial Repair Efficacy of Mesenchymal Stem Cells by Stimulating the Expression of Angiogenic Growth Factors and Gap Junction Protein. Adv. Funct. Mater. 2015, 25, 2590-2600.

(18) Srivastava, S. K.; Yadav, V. G. Bionic Manufacturing: Towards Cyborg Cells and Sentient Microbots. Trends Biotechnol. 2018, 36, 483-487.

(19) Fakhrullin, R. F.; Zamaleeva, A. I.; Minullina, R. T.; Konnova, S. A.; Paunov, V. N. Cyborg Cells: Functionalisation of Living Cells with Polymers and Nanomaterials. Chem. Soc. Rev. 2012, 41, 41894206.

(20) Niu, J.; Lunn, D. J.; Pusuluri, A.; Yoo, J. I.; O’Malley, M. A.; Mitragotri, S.; Soh, H. T.; Hawker, C. J. Engineering Live Cell Surfaces with Functional Polymers via Cytocompatible Controlled Radical Polymerization. Nat. Chem. 2017, 9, 537-545.

(21) Konnova, S. A.; Lvov, Y. M.; Fakhrullin, R. F. Nanoshell Assembly for Magnet-Responsive Oil-Degrading Bacteria. Langmuir 2016, 32, 12552-12558.

(22) Stanton, M. M.; Park, B. W.; Miguel-Lopez, A.; Ma, X.; Sitti, M.; Sanchez, S. Biohybrid Microtube Swimmers Driven by Single Captured Bacteria. Small 2017, 13, No. 1603679.

(23) Rogers, J. A. Electronics for the Human Body. Jama 2015, 313, $561-562$

(24) Kong, Y. L.; Gupta, M. K.; Johnson, B. N.; McAlpine, M. C. 3D Printed Bionic Nanodevices. Nano Today 2016, 11, 330-350.

(25) Mannoor, M. S.; Jiang, Z.; James, T.; Kong, Y. L.; Malatesta, K. A.; Soboyejo, W. O.; Verma, N.; Gracias, D. H.; McAlpine, M. C. 3D Printed Bionic Ears. Nano Lett. 2013, 13, 2634-2639.

(26) Gao, W.; Feng, X.; Pei, A.; Kane, C. R.; Tam, R.; Hennessy, C.; Wang, J. Bioinspired Helical Microswimmers Based on Vascular Plants. Nano Lett. 2014, 14, 305-310.

(27) Wu, S.; Zhou, Z.; Xu, L.; Su, B.; Fang, Q. Integrating Bipolar Electrochemistry and Electrochemiluminescence Imaging with Microdroplets for Chemical Analysis. Biosens. Bioelectron. 2014, 53, 148153.

(28) Loget, G.; Roche, J.; Kuhn, A. True Bulk Synthesis of Janus Objects by Bipolar Electrochemistry. Adv. Mater. 2012, 24, 511151165144

(29) Koizumi, Y.; Shida, N.; Ohira, M.; Nishiyama, H.; Tomita, I.; Inagi, S. Electropolymerization on Wireless Electrodes towards Conducting Polymer Microfibre Networks. Nat. Commun. 2016, 7, No. 10404.

(30) Wood, M.; Zhang, B. Bipolar Electrochemical Method for Dynamic In Situ Control of Single Metal Nanowire Growth. ACS Nano 2015, 9, 2454-2464.

(31) Bradley, J. C.; Chen, H. M.; Crawford, J.; Eckert, J.; Ernazarova, K.; Kurzeja, T.; Lin, M. D.; McGee, M.; Nadler, W.; Stephens, S. G. Creating Electrical Contacts between Metal Particles using Directed Electrochemical Growth. Nature 1997, 389, 268-271.

(32) Woodson, M.; Liu, J. Guided Growth of Nanoscale Conducting Polymer Structures on Surface-Functionalized Nanopatterns. J. Am. Chem. Soc. 2006, 128, 3760-3763.

(33) Kawasaki, J. K.; Arnold, C. B. Synthesis of Platinum Dendrites and Nanowires via Directed Electrochemical Nanowire Assembly. Nano Lett. 2011, 11, 781-785.

(34) Nikolaev, K. G.; Ermakov, S. S.; Offenhausser, A.; Mourzina, Y. Nonenzymatic Determination of Glucose on Electrodes Prepared by Directed Electrochemical Nanowire Assembly (DENA). J. Anal. Chem. 2017, 72, 371-374.

(35) Ramanathan, K.; Bangar, M. A.; Yun, M.; Chen, W.; Myung, N. V.; Mulchandani, A. Bioaffinity Sensing using Biologically Function- 
alized Conducting-Polymer Nanowire. J. Am. Chem. Soc. 2005, 127, 496-497.

(36) Thapa, P. S.; Yu, D. J.; Wicksted, J. P.; Hadwiger, J. A.; Barisci, J. N.; Baughman, R. H.; Flanders, B. N. Directional Growth of Polypyrrole and Polythiophene Wires. Appl. Phys. Lett. 2009, 94, No. 033104.

(37) Vaithilingam, J.; Saleh, E.; Korner, L.; Wildman, R. D.; Hague, R. J. M.; Leach, R. K.; Tuck, C. J. 3-Dimensional Inkjet Printing of Macro Structures from Silver Nanoparticles. Mater. Des. 2018, 139, $81-88$.

(38) Cheng, C.; Gonela, R. K.; Gu, Q.; Haynie, D. T. Self-Assembly of Metallic Nanowires from Aqueous Solution. Nano Lett. 2005, 5, $175-178$.

(39) Montal, M.; Mueller, P. Formation of Bimolecular Membranes from Lipid Monolayers and a Study of Their Electrical Properties. Proc. Natl. Acad. Sci. U.S.A. 1972, 69, 3561-3566.

(40) He, Y. F.; Zhang, F.; Saleh, E.; Vaithilingam, J.; Aboulkhair, N.; Begines, B.; Tuck, C. J.; Hague, R. J. M.; Ashcroft, I. A.; Wildman, R. D. A Tripropylene Glycol Diacrylate-based Polymeric Support Ink for Material Jetting. Addit. Manuf. 2017, 16, 153-161. 